\title{
IDENTIFICATION AND EVALUATION OF CORONAVIRUS REPLICASE INHIBITORS USING A REPLICON CELL LINE
}

\author{
Elke Scandella, Klara K. Eriksson, Tobias Hertzig, Christian Drosten, \\ Lili Chen, Chunshan Gui, Xiaomin Luo, Jianhua Shen, Xu Shen, \\ Stuart G. Siddell, Burkhard Ludewig, Hualiang Jiang, Stephan Günther, \\ and Volker Thiel*
}

\section{INTRODUCTION}

In order to provide a rapid and safe assay for the identification and evaluation of coronavirus replicase inhibitors, we have generated a non-cytopathic, selectable replicon RNA (based on human coronavirus $229 \mathrm{E}$ [HCoV-229E]) that can be stably maintained in eukaryotic cells. ${ }^{1}$ Stable, replicon RNA-containing cell lines that express green fluorescent protein (GFP) as a marker for coronavirus replication have been used to test the inhibitory effect of several compounds that are currently being assessed for SARS treatment or have been predicted to target replicative proteins. Amongst those, interferonalpha and cinanserin displayed the strongest inhibitory activities. Interestingly, cinanserin is a well-characterized serotonin antagonist that has already undergone preliminary clinical testing in humans in the $1960 \mathrm{~s}^{2-4}$ Cinanserin has been identified as candidate inhibitor of the SARS-CoV 3C-like proteinase by virtual screening of a database containing structural information of more than 8,000 existing drugs using a docking approach for potential binding to the SARS-CoV 3C-like proteinase. ${ }^{5}$ Subsequently, binding of cinanserin to bacterially expressed SARS-CoV 3C-like proteinase and inhibition of its enzymatic activity was demonstrated experimentally. Antiviral activity of cinaserin was further evaluated in two tissue culture-based assays. First, we have used our safe replicon assay and could demonstrate a strong inhibitory activity of cinanserin. Second, we could demonstrate a strong inhibition (up to 4 log reduction of virus RNA and infectious particles) of SARS-CoV and $\mathrm{HCoV}-229 \mathrm{E}$ replication in tissue culture.

\footnotetext{
*Elke Scandella, Klara K. Eriksson, Burkhard Ludewig, Volker Thiel, Kantonal Hospital St. Gallen, 9007 St. Gallen, Switzerland. Tobias Hertzig, University of Würzburg, Germany. Christian Drosten, Stephan Günther, Bernhard-Nocht-Institute, Hamburg, Germany. Lili Chen, Chunshan Gui, Xiaomin Luo, Jianhua Shen, Xu Shen, Hualiang Jiang, Drug Discovery Center, Shanghai, China. Stuart G. Siddell, University of Bristol, United Kingdom.
} 
These findings demonstrate that the old drug cinanserin is a potent inhibitor of SARS$\mathrm{CoV}$ replication and illustrate the value of non-infectious coronavirus replicon RNAs for the biosafe screening and evaluation of candidate replicase inhibitors.

\section{RESULTS}

\subsection{Generation of Coronavirus Replicon Cell Lines}

The recent development of reverse genetic systems for coronaviruses enables the generation of autonomously replicating RNAs based on a recombinant coronavirus genome. To construct a coronavirus-based replicon RNA we made use of our reverse genetic system for HCoV-229E. ${ }^{6}$ The full-length $\mathrm{HCoV}-229 \mathrm{E}$ cDNA, that has been cloned into a vaccinia virus vector, was modified by vaccinia virus-mediated recombination. We have introduced the gene for a selectable marker (neo) into the $\mathrm{HCoV}$ $229 \mathrm{E}$ replicase gene and we have replaced three structural genes (S, E, and M), and two accessory genes ( $4 \mathrm{a}$ and $4 \mathrm{~b}$ ), by a reporter gene encoding green fluorescent protein (GFP). A stable cell line, designated BHK-Rep-1, that contains the replicon RNA has been selected using G418. This cell line displays green fluorescence as a marker for coronavirus replication (Figure 1).

The HCoV-229E-based replicon cell line, BHK-Rep-1, can be used to assess inhibitory effects of candidate replicase inhibitors without the need to propagate infectious virus. ${ }^{1}$ Graded doses of compounds are added to BHK-Rep-1 cells and after three days reporter gene expression levels are determined using fluorescence microscopy and FACS analysis. Decreasing reporter gene expression indicates the antiviral activity of a particular compound. In parallel the cytotoxicity of candidate compounds are determined on parental BHK-21 cells.

Several compounds have been tested for antiviral activity. Interferon- $\alpha$ (IFN- $\alpha$ ) reduced the level of GFP expression and the overall number of green fluorescent cells (Figure 2). Titration of IFN- $\alpha$ showed that the inhibition of coronavirus replication was
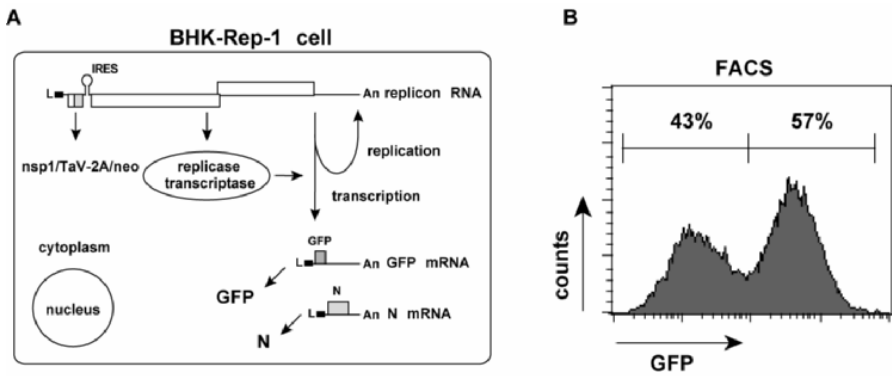

Figure 1. HCoV-229E replicon cell line. (A) The predicted replicon RNA-mediated gene exptession in BHKRep- 1 cells is illustrated. (B) FACS analysis of GFP expression in BHK-Rep-1 cells. 
A

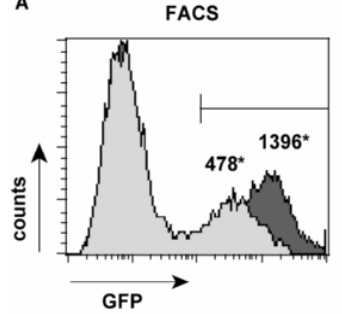

B

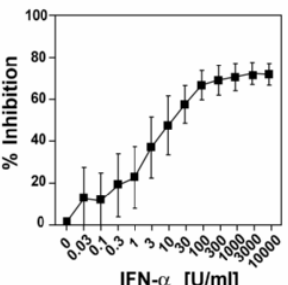

c

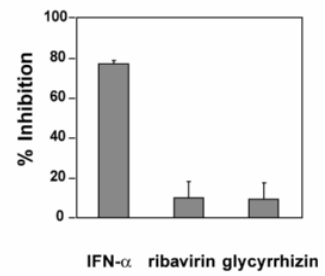

Figure 2. Inhibition of coronavirus replication. (A) FACS analysis of untreated (dark gray) and IFN- $\alpha$-treated (light gray, 10,000 U/ml) BHK-Rep-1 cells. Indicated values represent the mean fluorescence intensity of gated (bar) cells. (B) Inhibition of GFP expression of IFN- $\alpha$-treated BHK-Rep-1 cells. (C) Inhibition of GFP expression of IFN- $\alpha$-treated $(10,000 \mathrm{U} / \mathrm{ml})$, ribavirin-treated $(300 \mu \mathrm{g} / \mathrm{ml})$, and glycyrrhizin-treated $(3000$ $\mu \mathrm{g} / \mathrm{ml})$ BHK-Rep-1 cells.

dose-dependent and half-maximal inhibition could be achieved with only $10 \mathrm{U} / \mathrm{ml}$. In contrast, the maximal inhibitory effects of ribavirin and glycyrrhizin did not exceed $10 \%$. Our data indicate that IFN- $\alpha$ appears to represent a promising candidate for the inhibition of coronavirus replicase function and furthermore that the inhibition of coronavirus replication can be monitored using coronavirus replicon RNA-containing cell lines.

\subsection{Identification and Evaluation of Cinanserin}

One of the main targets for antiviral intervention is the coronavirus 3C-like proteinase $\left(3 \mathrm{CL}^{\mathrm{pro}}\right)$. The $3 \mathrm{CL}^{\text {pro }}$ is responsible for the proteolytic release of replicative proteins from their replicase precursor polyproteins and is, therefore, considered indispensable for virus replication. ${ }^{7}$ In order to identify candidate $3 \mathrm{CL}^{\text {pro }}$ inhibitors, a homology model of the binding pocket of the SARS-CoV $3 \mathrm{CL}^{\text {pro }}$, based on crystallographic structures of TGEV and HCoV229E proteinases, ${ }^{8}$ was used as target for screening in silico. Screened compounds were derived from the Comprehensive Medical Chemistry database of Mol. Design Limited, which contains pharmacological and structural information of $>8000$ compounds used or evaluated as therapeutic agents in humans. Cinanserin, a well-characterized serotonin antagonist, showed a high score in the screening and was chosen for experimental testings. First, the SARS-CoV $3 \mathrm{CL}^{\text {pro }}$ was expressed in E. coli, and the purified enzyme was used to demonstrate (i) binding of cinanserin to $3 \mathrm{CL}^{\text {pro }}$, and (ii), inhibition of $3 \mathrm{CL}^{\text {pro }}$ by cinanserin in vitro. Maximum inhibition was observed at concentrations of 50-100 $\mu \mathrm{M}$ (data not shown). Second, we have used the BHK-Rep-1 cells to assess the inhibitory activity of cinanserin in tissue culture. BHK-Rep-1 cells were treated for 3 days with cinanserin, and the expression of GFP was analyzed by FACS and fluorescence microscopy. The number of green fluorescent cells was greatly reduced at a concentration of $30 \mu \mathrm{g} / \mathrm{ml}$ (Figure 3a). This indicates that cinanserin is able to enter the target cell and inhibit coronavirus replicase function. Finally, Vero cells were infected with SARS-CoV and treated with cinanserin $(0-50 \mu \mathrm{g} / \mathrm{ml})$. Two days postinfection, virus RNA in the tissue culture supernatant was analyzed by real-time RT-PCR (Figure 3b). In addition the titer of infectious particles in the supernatant was measured by immunofocus assay (Figure 3c). Our results clearly 


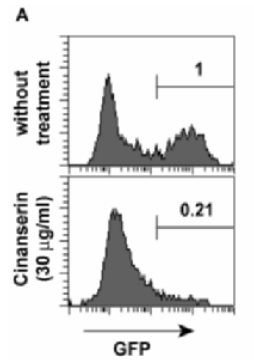

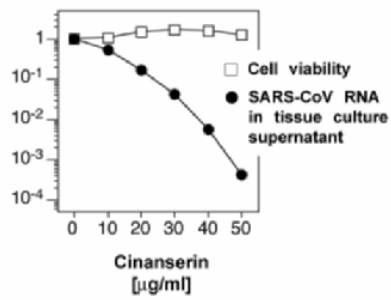

c

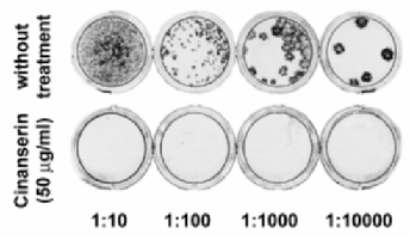

Figure 3. Inhibition of coronavirus replication by cinanserin. (A) Inhibition of GFP expression in BHK-Rep-1 cells by cinanserin $(30 \mu \mathrm{g} / \mathrm{ml})$. The number of GFP-expressing untreated cells was set as 1 . (B) Reduction of SARS-CoV RNA concentration in cell culture supernatant. Vero cells were infected with SARS-CoV (moi 0.01), and virus RNA concentration was measured by real-time PCR after 2 days. Cell viability was determined by the MTT test. The virus RNA concentration of untreated cells and the corresponding cell viability value were defined as 1. (C) Reduction of SARS-CoV infectious particles in supernatant. Supernatants of cinanserin-treated $(50 \mu \mathrm{g} / \mathrm{ml})$ or untreated infected cells were harvested 2 days postinfection (moi 0.01$)$ and the numbers of infectious particles were determined by immunofocus assay. Cell culture wells inoculated with dilutions of supernatant are shown.

demonstrate that both viral RNA in the tissue culture supernatant and infectious particles were greatly reduced by up to $4 \log$ units.

\section{CONCLUSIONS}

In this study we have used the concept of selectable coronavirus replicon RNAs to assess the inhibitory activities of candidate coronavirus replicase inhibitors. We have shown that stable replicon RNA-containing cell lines provide a rapid assay for the identification and evaluation of coronavirus replicase inhibitors. Although the replicon RNA used in this study is based on HCoV-229E, our concept is also applicable to the generation of SARS-CoV replicon RNAs. Stable cell lines containing SARS-CoV replicon RNAs will enable the biosafe screening and evaluation of SARS-CoV replicase inhibitors without the need to grow infectious virus.

\section{ACKNOWLEDGMENTS}

This work was supported by the Swiss National Science Foundation, the Gebert-Rüf Foundation, Switzerland, and the Deutsche Forschungsgemeinschaft.

\section{REFERENCES}

1. T. Hertzig, E. Scandella, B. Schelle, J. Ziebuhr, S. G. Siddell, B. Ludewig, and V. Thiel, Rapid identification of coronavirus replicase inhibitors using a selectable replicon RNA, J. Gen. Virol. 85, 1717-1725 (2004).

2. B. Rubin, J. J. Piala, J. C. Burke, and B. N. Craver, A new, potent and specific serotonin inhibitor (Sq 10,643) 2'-(3-dimethylaminopropylthio) cin-namanilide hydrochloride: antoserotonin activity on uterus and on gastrointestinal, vascular, and respiratory systems of animals, Arch. Int. Pharmacodyn. Ther. 152, 132143 (1964). 
3. D. M. Gallant and M. P. Bishop, Cinanserin (SQ. 10,643): a preliminary evaluation in chronic schizophrenic patients, Curr. Ther. Res. Clin. Exp. 10, 461-463 (1968).

4. T. M. Itil, N. Polvan, and J. M. Holden, Clinical and electroencephalographic effects of cinanserin in schizophrenic and manic patients, Dis. Nerv. Syst. 32, 193-200 (1971).

5. L. Chen, C. Gui, X. Luo, et al., Cinanserin is an inhibitor of the 3C-like proteinase of severe acute respiratory syndrome coronavirus and strongly reduces virus replication in vitro, J. Virol. 79, 7095-7103 (2005).

6. V. Thiel, J. Herold, B. Schelle, and S. G. Siddell, Infectious RNA transcribed in vitro from a cDNA copy of the human coronavirus genome cloned in vaccinia virus, J. Gen. Virol. 82, 1273-1281 (2001).

7. J. Ziebuhr, E. J. Snijder, and A. E. Gorbalenya, Virus-encoded proteinases and proteolytic processing in the Nidovirales, J. Gen. Virol. 81, 853-879 (2000).

8. K. Anand, J. Ziebuhr, P. Wadhwani, J. R. Mesters, and R. Hilgenfeld, Coronavirus main proteinase (3CLpro) structure: basis for design of anti-SARS drugs, Science 300, 1763-1767 (2003). 